\title{
RESISTÊNCIA AO IMPACTO DE COMPÓSITOS EPOXÍDICOS REFORÇADOS COM TECIDO DE RAMI SOB VÁCUO *
}

\author{
Caroline Gomes de Oliveira ${ }^{1}$ \\ Janine Feitosa de Deus ${ }^{2}$ \\ Felipe Perissé Duarte Lopes ${ }^{3}$ \\ Frederico Muylaert Margem ${ }^{4}$ \\ Sérgio Neves Monteiro ${ }^{5}$ \\ Lucas de Almeida Pontes ${ }^{6}$
}

\section{Resumo}

Os impactos causados pela exploração dos recursos naturais pelo homem geram uma crescente inquietação em relação futuro do planeta a longo prazo. Assim, as fibras naturais vegetais ganham destaque como material de reforço por suas boas propriedades mecânicas, baixo custo e por serem biodegradáveis. Umas das fibras que mais ganhou destaque nas últimas décadas é a fibra de rami, objeto de inúmeros estudos. Neste trabalho, analisou-se o efeitodo tecido produzido com a fibra de rami como reforço para a matriz epóxi sob esforços de impacto Charpy e Izod. A metodologia utilizada foi a produção de placas laminadas sob afeito de vácuo. Os resultados mostram notável diferença na resistência ao impacto do compósito comparado à matriz sem reforço.

Palavras-chave:Impacto Izod; Impacto Charpy; Tecido de Rami; Fibras Naturais

\section{IMPACT RESISTANCE OF EPOXYDIC COMPOSITES REINFORCED WITH RAMI FABRIC UNDER VACUUM}

\begin{abstract}
The impacts caused by the exploitation of natural resources by man, generates a growing uneasiness regarding the planet future in a long term. So, the natural vegetal fibers stand out as analternative reinforcement material for their good mechanical properties, low cost and for being biodegradable. One of the natural fibers that has gained more prominence in last decades is the rami fiber, object of numerous studies. In this work, it was investigated the effect of ramie fabric as reinforcement for the epoxy matrix under Charpy and Izod impact tests. The methodology used was the production of laminated plates under vacuum. The results show a remarkable difference in the impact resistance of the composite compared to the non-reinforced matrix.

Keywords: Izod Impact;Charpy Impact; Ramie Fabric; Natural Fibers

1 Metallurgical and Materials Engineering, Laboratory of Advanced Materials, State University of the Northern Rio de Janeiro Darcy Ribeiro, Campos dos Goytacazes, RJ - Brazil

2 M.Sc. Metallurgical and Materials Engineering, Doctor Student, Laboratory of Advanced Materials, State University of the Northern Rio de Janeiro Darcy Ribeiro, Campos dos Goytacazes, RJ - Brazil

3 D.Sc. Metallurgical and Materials Engineering, Colaborator, Laboratory of Advanced Materials, State University of the Northern Rio de Janeiro Darcy Ribeiro, Campos dos Goytacazes, RJ - Brazil

4 D.Sc. Metallurgical and Materials Engineering, Assistant Professor, Center of Science and Engineering, Redentor University, Itaperuna, RJ - Brazil.

5 PhD Metallurgical and Materials Engineering, PhD, Main Professor, Laboratory of Materials and Metallurgy, Military Institute of Technology, Rio de Janeiro, RJ - Brazil.

6 Student, UENF, RJ, Brazil.
\end{abstract}




\section{INTRODUÇÃO}

Com o desenvolvimento tecnológico a demanda por materiais com baixos custos e que não prejudiquem o meio ambiente vem crescendo. A tendência atual da nossa sociedade aponta para a busca de materiais ecológicos, que podem contribuir para reduzir as emissões de $\mathrm{CO} 2$, principal causa do efeito estufa. Seguindo esse paradigma, os pesquisadores mostraram interesse na reciclagem de materiais, uso de resíduos e uso de materiais naturais [1,2].

As aplicações de fibras lignocelulósicas naturais obtidas a partir de vegetais e plantas são valorizadas na engenharia como uma alternativa ecológica para substituir materiais não recicláveis que resultam em alto consumo de energia, como, por exemplo, compósitos de fibra de vidro [3]. De fato, o mundo mostrou uma alta tendência a substituir a fibra de vidro por fibras naturais em aplicações tipicamente dominadas pelos materiais compósitos [4,5]. O uso de fibras naturais revela várias vantagens em relação à fibra de vidro, como por exemplo, o custo mais barato, e o fato das fibras naturais serem mais leves e não abrasivas para equipamentos utilizados em seu processamento. Algumas fibras sintéticas (incluindo a de vidro) apresentam um risco para a saúde quando inaladas e sua produção está associada a emissões de $\mathrm{CO} 2$, a principal causa do efeito estufa e responsável pelo aquecimento global. Nenhuma destas falhas aplica-se às fibras lignocelulósicas extraídas de plantas, que são renováveis, biodegradáveis e não contribuem em emissões médias de $\mathrm{CO} 2$ pois absorvem este gás ao longo de seu ciclo de vida [6].

As fibras naturais lignocelulóticas de rami tem sido amplamente investigadas em vários estudos, como reforço em compósitos de poliméricos. Esses compósitos apresentaram altas propriedades mecânicas [7-9]. Buscando melhorar o desempenho mecânico destes materiais naturais, o uso de tecidos naturais tem sido estudado como mais uma alternativa de reforço para compósitos poliméricos. Algumas de suas vantagens são a possibilidade de reforçar a matriz em mais de uma direção simultaneamente e a possibilidade de introduzir uma fração maior de fibras naturais, uma vez que o tecido é mais compacto do que as fibras alinhadas $[10,11]$.

Considerando-se esses fatores, é válido investir em materiais reforçados com tecido de rami, uma vez que esta constituiu uma das fibras naturais mais resistentes estudadas [12]. O objetivo deste estudo foi investigar a resistência aos impactos Charpy e Izod de compósitos de matriz de epóxi reforçados com tecido de rami.

\section{MATERIAIS E MÉTODOS}

O tecido utilizado neste trabalho foi importado da companhia chinesa Rose Natural Healthy Items Wholesale, Fig. 1 (a). Sua densidade foi verificada pelo Método de Arquimedes e o valor obtido foi de $245 \mathrm{~g} / \mathrm{m} 2$. Foram produzidas placas de compósito que foram cortadas nas medidas do corpo de prova para os ensaios de impacto Charpy e Izod.

Para a produção das placas laminadas, camadas de tecido foram embebidas em resina epóxi (DEGEBA/TETA) e empilhadas dentro do molde (Fig. 1b). Para a aplicação do vácuo, um filme é sobreposto ao molde, tendo suas laterais vedadas com fita adesiva dupla face de alta aderência (tacky tape). O sistema é ligado a uma bomba de vácuo através de uma fina mangueira, retirando todo o ar do sistema, a fim de evitar a formação de bolhas. 

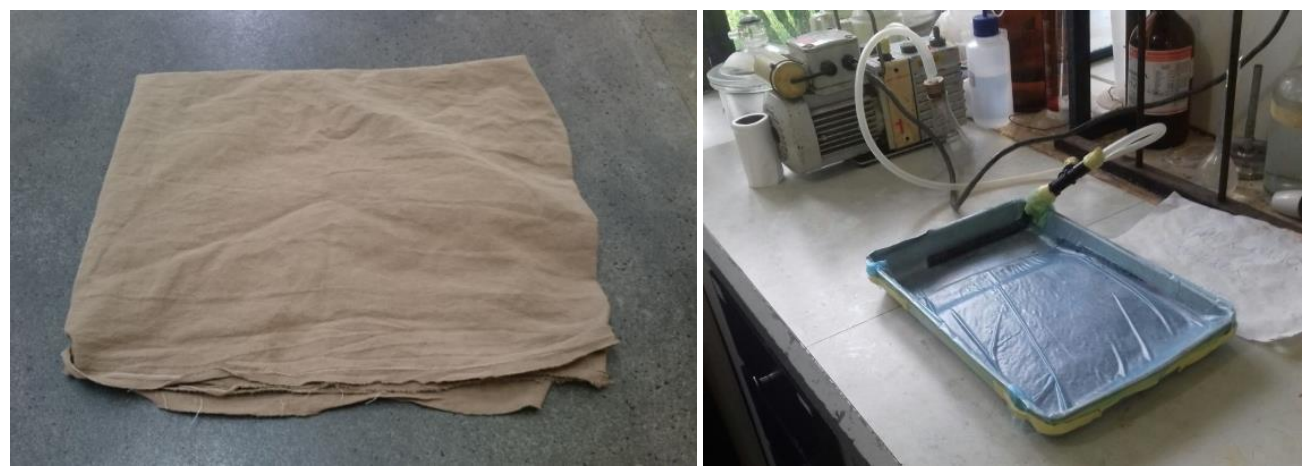

Figura 1: Tecido de rami como recebido no laboratório (a) e sitema de vácuo (b).

Após cura de $24 \mathrm{~h}$ em temperatura ambiente, a placa foi cortada em corpos de prova medindo 63,5 x 12,7 x 10mm (ASTM D256, [13]) para o ensaio de Impacto Izod e medindo $127 \times 12,7 \times 10 \mathrm{~mm}$ para o ensaio de Impacto Charpy (ASTM D6110 [14]). Os corpos de prova foram ensaiados em um Pêndulo Pantec, nas configurações Charpy e Izod.

\section{RESULTADOS E DISCUSSÃO}

\subsection{Impacto Charpy}

A Fig. 2 mostra o copo de prova após o ensaio de impacto Charpy

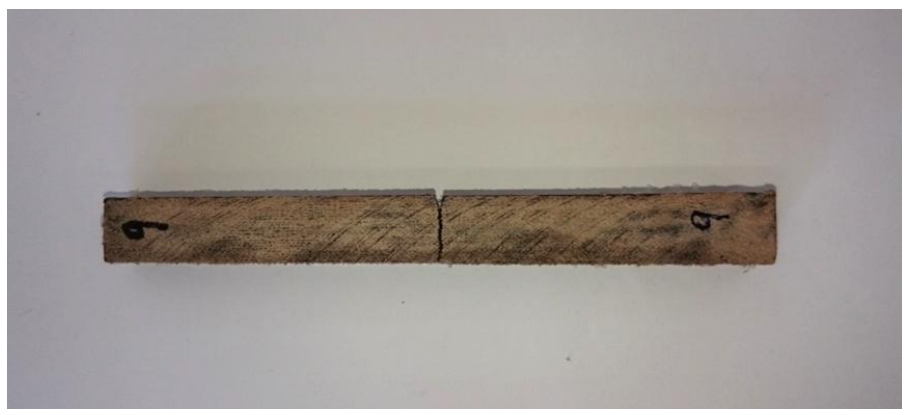

Figura 2: Corpo de prova após o ensaio de impacto Charpy.

É possível observar que a fratura ocorreu de maneira transversal à sua espessura, caracterizando uma fratura frágil. Além disso, pode-se observar que o rompimento do corpo de prova foi completo, sem participação do tecido. Isso indica a ausência do efeito pullout, em que ocorre o escorregamento do reforço para fora da matriz antes do rompimento das fibras, um dos fatores responsáveis pela absorção de energia [15].

A energia média absorvida pelo compósito até a ruptura foi de $249,56 \pm 12,66 \mathrm{~J} / \mathrm{m}$, enquanto a energia média absorvida pelo epóxi foi de $22,17 \pm 4,45 \mathrm{~J} / \mathrm{m}$. Com base nos resultados apresentados, foi possível elaborar um diagrama de variação de resistência ao impacto (Fig. 3), com o objetivo de evidenciar a amplitude do efeito de reforço introduzido pela presença do tecido. 


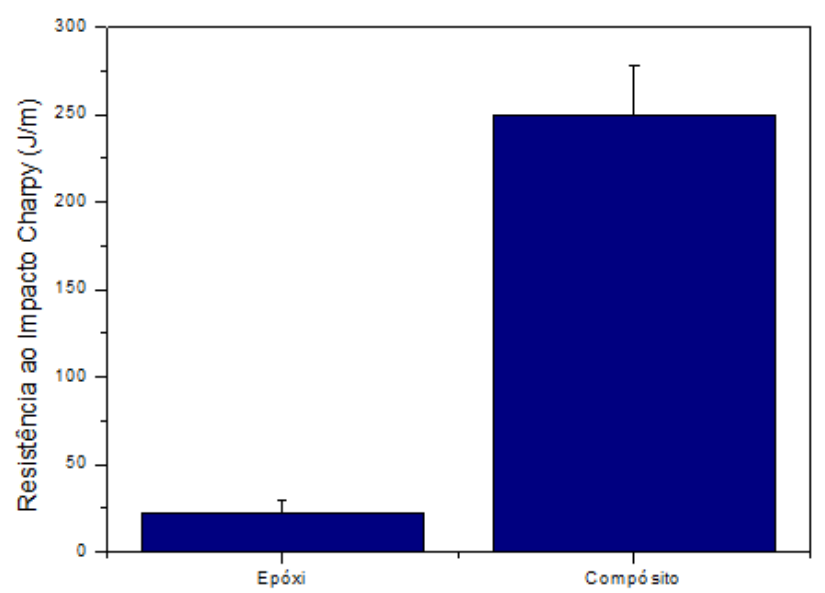

Figura 3: Variação da resistência ao impacto Charpy para o compósito formulado e a matriz epóxi.

A presença do tecido aumentou em mais de dez vezes a resistência do material ao impacto, comparado com a matriz epóxi.

Apesar das fibras naturais (incluindo a fibra de rami) apresentarem, de maneira geral, baixa adesão à matriz [16] o escorregamento neste caso não ocorre. Isso possivelmente se deve à configuração entrelaçada do tecido, que contribui para que este fique retido à matriz. Enquanto as fibras alinhadas à direção do esforço tendem a escorregar da matriz, as fibras transversais (a $\left.90^{\circ}\right)$ não sofrem esse efeito e ajudam a reter o tecido na matriz. Desta maneira, as fibras do tecido rompem sem que escorreguem da matriz.

A Fig. 4 mostra a micrografia da superfície de fratura do compósito após a realização do ensaio de impacto Charpy.
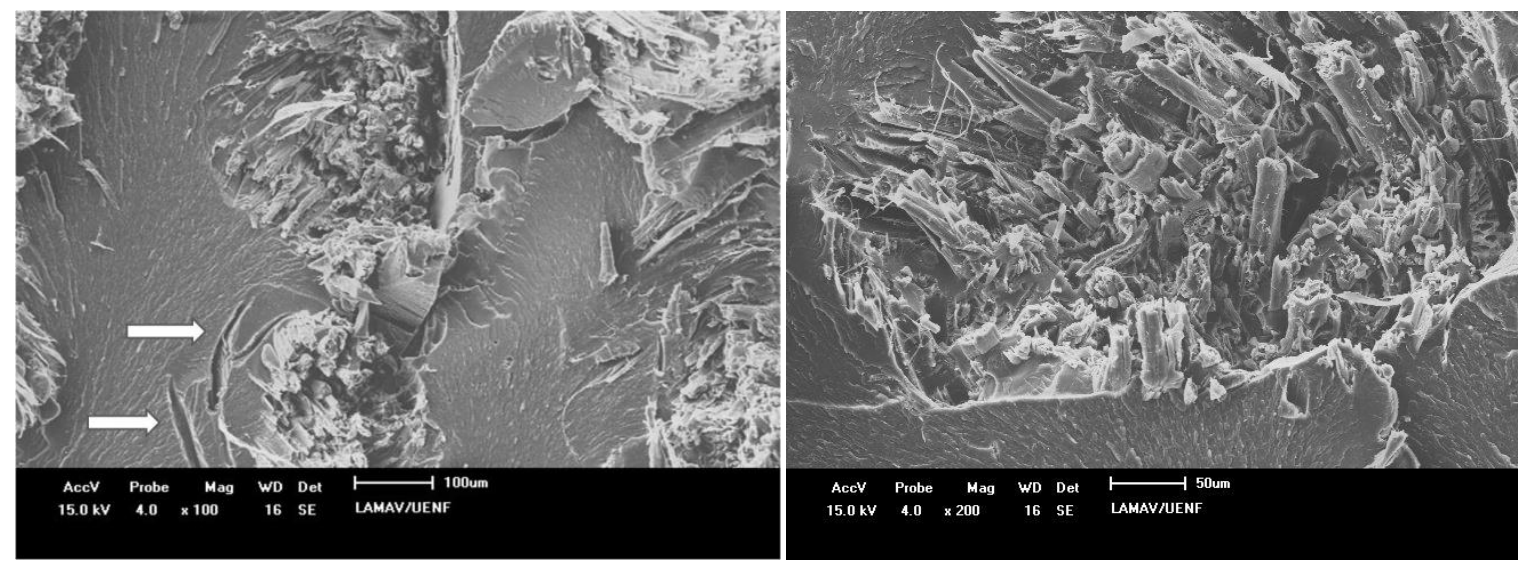

Figura 4: Micrografia da superfície de fratura do compósito reforçado com tecido de rami após o ensaio de impacto Charpy com aumento de100x (a) e 200x (b).

Na Fig. 4 (a) estão indicados os locais onde as trincas foram desviadas pelas fibras do tecido de rami. Na Figura 4 (b) pode-se observar que as fibras do tecido romperam aproximadamente paralelas ao ponto em que a matriz fraturou, o que evidencia que não ocorreu o efeito pull out. 


\subsection{Impacto Izod}

A fratura dos corpos de prova do ensaio de impacto Izod, assim como os de Charpy, apresentou fratura tipicamente frágil com alguns desvios e com total rompimento da matriz e do tecido, como mostrado na Figura 5.

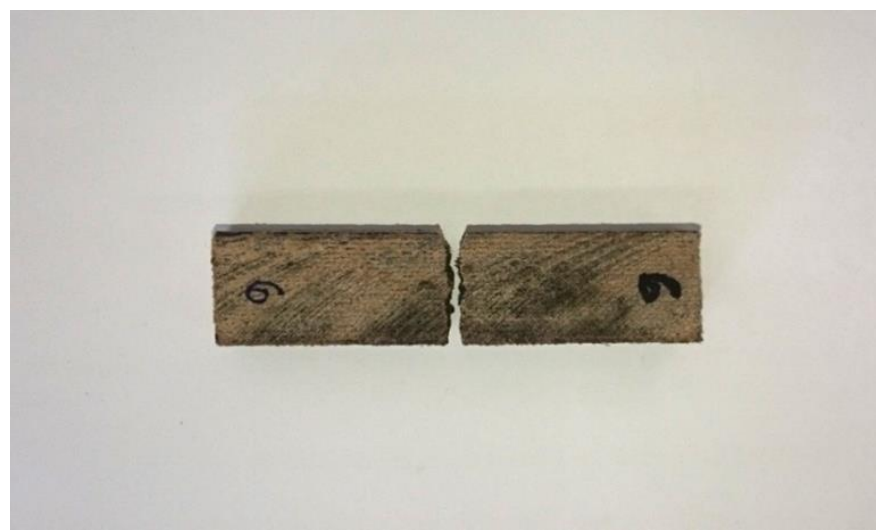

Figura 5: Corpo de prova após o ensaio de impacto Izod.

A completa fratura do material indica que não houve escorregamento significativo do tecido para fora da matriz, o que fica evidente na Fig. 6 (a), que mostra a micrografia da superfície de fratura do material após a realização do ensaio de impacto.
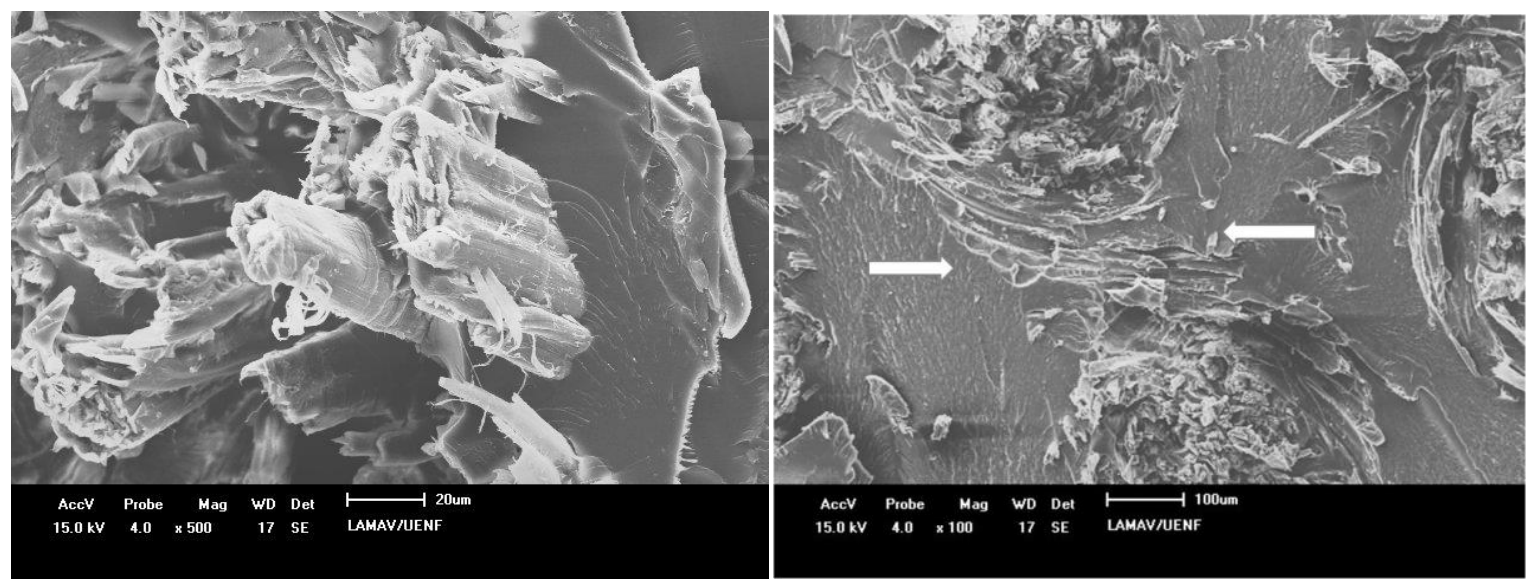

Figura 6: Micrografia da superfície de fratura do compósito após o ensaio de impacto Izod. Aumento de 500x (a) e 100x (b).

A Fig.6 (b) mostra outro ponto da superfície de fratura, onde é possível observar que, ao encontrar as fibras do tecido, a trinca sofre desvio. Também pode-se observar que as fibras do tecido rompem aproximadamente à mesma altura em que a matriz, confirmando, mais uma vez, que o escorregamento entre a matriz e a fibra não ocorre.

Apesar de não ter ocorrido escorregamento, processo em que há significativa absorção de energia, o aumento na resistência ao impacto foi considerável. 0 compósito reforçado com tecido de rami apresentou resistência média de 110,50 \pm $6,00 \mathrm{~J} / \mathrm{m}$ em contraste com 18,98 $\pm 4,21 \mathrm{~J} / \mathrm{m}$ da matriz epóxi. O aumento na resistência ao impacto do compósito foi cerca de cinco vezes a resistência da matriz epóxi sem reforço. Esse aumento fica evidenciado no diagrama de variação da resistência ao impacto Izod do compósito e da matriz epóxi (Fig. 7). 


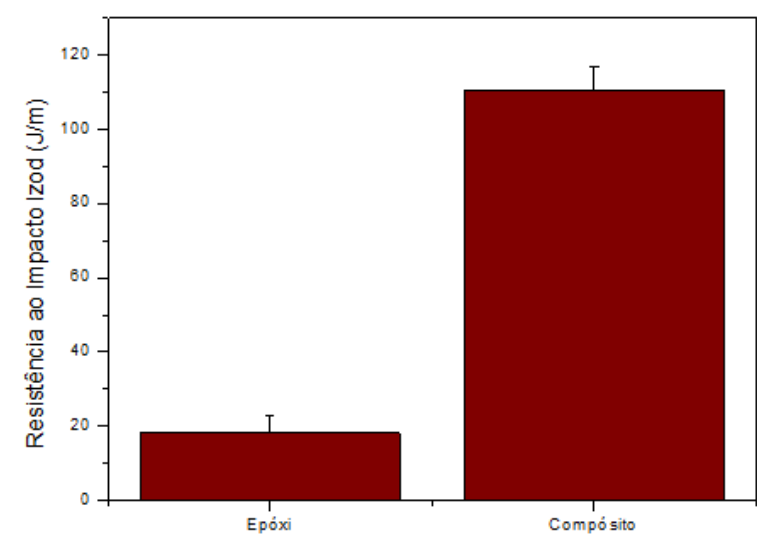

Figura 7: Variação da resistência ao impacto Izod do compósito formulado e da matriz epóxi.

A diferença na proporção do reforço nos dois tipos de ensaio provavelmente se deve à diferença na aplicação do esforço e, consequentemente, à diferença na resposta do material a dois esforços distintos. No entanto vale ressaltar que em ambos os casos a presença do tecido aumentou de maneira significativa a resistência da matrix epóxi ao impacto.

\section{CONCLUSÃO}

- A introdução do tecido de rami se mostrou efetiva no aumento da resistência da matriz epóxi ao impacto (Charpy e Izod), demonstrando um ganho mais elevado na resistência ao impacto Charpy.

- A adesão entre o tecido e a matriz é considerável e o efeito pull out não ocorre. As fibras do tecido servem como barreiras, desviando a trinca durante sua propagação.

- A metodologia de fabricação se mostrou eficaz, aumentando a interação entre as fibras naturais do tecido e a matriz epóxi e assegurando que as fibras consigam atuar como agentes de desvio da propagação de trincas. Com isso, levou a aumentos significativos da resistência ao impacto do material.

\section{AGRADECIMENTOS}

Os autores agradecem às agências de fomento: CNPq, CAPES e FAPERJ.

\section{REFERÊNCIAS}

[1] Chawla KK. CompositeMaterials, New York: Springer-Verlag (1993).

[2] Monteiro SN, Lopes FPD, Ferreira AS, Nascimento DCO Natural fiberpolymermatrixcomposites: cheaper, tougherandenvironmentallyfriendly, JOM, 61 (2009) 17-22.

[3] Wambua P, Ivens I, Verpoest I. Natural fibers: cantheyreplaceglassandfiberreinforcedplastics?. Comp. Sci. Technol. v. 63, p. 1259-1264, 2003. 
[4] Mohanty

AK,

Misra

M,

Hinrichsen,

G.Biofibers, biodegradablepolymersandbiocomposites: an overview. Macromolecular Mat. andEngineering, 276/277, p. 1-24, 2000.

[5] Monteiro SN, Lopes FPD, Ferreira AS, Nascimento DCO. Natural fiberpolymermatrixcomposites: cheaper, tougherandenvironmentallyfriendly. JOM, 61(1), pp. 17-22, 2009.

[6] Crocker J. Natural materialsinnovative natural composites. Materials Technology, 2-3 (2008) 174-178.

[7] Angelini LG, Lazzeri A, Levita G,Fontanelli D, Bozzi C. Ramie (Boehmerianivea (L.) Gaud.) and Spanish Broom (Spartiumjunceum L.) fibers for compositematerials: agronomicalaspects, morphologyandmechanicalproperties, Industrial CropsandProducts, v.11(2-3), p.145-161, 2000.

[8] Shihong L, Benlian Z, Qiyun Z, Xianrong B. A new kindofsuper-hybridcomposite material for civil use - ramiefibre/Al, Composites, v. 25(3), p. 225-228,1994

[9] Monteiro SN, Satyanarayana KG, Lopes FPD.High strength natural fibers for improvedpolymermatrixcomposites, Materials Science Forum, 638-642 (2010) 961-966.

[10] Mohanty AK, Khan MA, Hinrichsen G. InfluenceofChemicalSurfaceModificationonthePropertiesofBiodegradableJuteFabr ics-PolyesterAmideComposites. Composites: Part A, v. 31, p. 143-150, 2000.

[11] Lei W, Wen-Guang L, Ren C.Effectof volume fractionoframieclothonphysicalandmechanicalpropertiesoframiecloth/UP resincomposite, TransactionsofNonferrousMetalsSocietyof China, v. 16(2), p. 474-477, 2006

[12] Giacomini NP. Compósitos reforçados com fibras naturais para a indústria automobilística, Dissertação de Mestrado, USP - Universidade de São Paulo, São Carlos-SP, p. 21, 2003.

[13] ASTM - American Society for Testing Materials. ASTM D256 - 02 - Standard Test Methods for Determining the Izod Pendulum Impact Resistance of Plastics. Annual Book of ASTM, 2002.

[14] ASTM - American Society for Testing Materials. ASTM D6110 - 04 - Standard Test Methods for Determining the Charpy Pendulum Impact Resistance of Notched Specimens of Plastics. Annual Book of ASTM, 2004.

[15] Ashby M, Shercliff H, Cebon D. Materials - Engineering, Science, Processingand Design, Elsevier, Burlington - MA (2007).

[16] Neto FL, Pardini LC. Compósitos Estruturais, Edgard Blücher, São Paulo - SP (2006). 